\title{
15q11.2 microdeletion syndrome
}

INSERM

\section{Source}

INSERM. (1999). Orphanet: an online rare disease and orphan drug data base. 15911.2 microdeletion syndrome. ORPHA:261183

$15 q 11.2$ microdeletion syndrome is a rare partial autosomal monosomy with a variable phenotypic expression and reduced penetrance associated with an increased susceptibility to neuropsychiatric or neurodevelopmental disorders including delayed psychomotor development, speech delay, autism spectrum disorder, attention deficithyperactivity disorder, obsessive-compulsive disorder, epilepsy or seizures. It may also include mild non-specific dysmorphic features (such as dysplastic ears, broad forehead, hypertelorism), cleft palate, neurological and neuroimaging abnormalities (such as ataxia and muscular hypotonia). 